\title{
Ontoepistemologías del aborto en Ecuador: reflexiones críticas y bioéticas
}

[Cómo citar este artículo: Escalona, D., Pérez, M., Delgado, H. Ontoepistemologías del aborto en Ecuador: reflexiones críticas y bioéticas. Práctica Familiar Rural. 2019 noviembre; 4(3).]

Dayamy Escalona León[a], Melissa Pérez Acosta[b], Hishochy Delgado Mendoza[a, c]

a. la Pontificia Universidad Católica del Ecuador sede Santo Domingo b. Estudiante de Medicina en la Universidad San Francisco de Quito c. Instituto Superior Tecnológico Japón

DOI: $\underline{\text { https://doi.org/10.23936/pfr.v4i2.93 }}$

Recibido: 22/08/2019 Aprobado: 29/10/2019

\section{Resumen}

El aborto constituye uno de los temas más candentes de la actualidad en cuestiones de salud desde la dimensión bioética. Su estudio ha devenido en inquietante misión para estudiantes, profesionales y demás miembros de la comunidad médica, entre otros interesados, por su impacto psicosocial, mórbido y cultural. El propósito de este artículo-ensayo es realizar una reflexión crítica sobre el aborto en el Ecuador y su impacto desde el punto de vista bioético. A través de este texto se busca ampliar las nociones del lector respecto al aborto y llegar a cuestionarnos: ¿cómo fortalecer juicios bioéticos respecto al aborto?, ¿cuáles son los beneficios, prejuicios y contraindicaciones de este?, ¿se respeta o no la autonomía y beneficencia del paciente? y ¿cómo esto podría afectar la vida de los mismo?

Palabras Claves: Aborto, salud, autonomía, bioética, beneficencia, vida

Ontoepistemologies of abortion in Ecuador: critical and bioethical reflections

\section{Abstract}

Abortion is one of the hottest topics of current health issues from the bioethical dimension. His study has become a disturbing mission for students, professionals and other members of the medical community, among others, for its psychosocial, morbid and cultural impact. The purpose of this articleessay is to make a critical reflection on abortion in Ecuador and its impact from the bioethical point of view. Through this text, we seek to broaden the 
reader's notions regarding abortion and to question ourselves: how to strengthen bioethical judgments regarding abortion? What are its benefits, prejudices and contraindications? Is autonomy respected or not? and patient benefit? And how could this affect their lives?

Keywords: Abortion, health, autonomy, bioethics, beneficence, life

\section{INTRODUCCIÓN}

\section{Abriendo caminos}

En ciencias de la salud, debido a las fuertes incidencias de la globalización cultural y del pensamiento posmoderno neoliberal a escala mundial, se han incrementado los cabos sueltos respecto a temas muy polémicos que ameritan discutir y resolver. Un ejemplo de ellos es el aborto. En este trabajo abordaremos -desde la reflexión crítica- dicho tema con sus controversias. Sobre todo, aquellos dilemas que genera el aborto inducido.

Las políticas, legislaciones y filosofías para tratar al abordo son diversas, cada país regula esta situación de acuerdo a lo establecido en sus Constituciones. Por ahora nos interesa reflexionar críticamente sobre el aborto en Ecuador; país donde vivimos y estudiamos medicina con aspiraciones a la pertinencia bioética. $Y$ es precisamente la Bioética el principio rector, el criterio dominante del presente ensayo; el mismo no fue elegido al azar, tiene una tesis irrefutable: los debates, los dilemas, las controversias que han provocado movimientos de lucha sobre el aborto en Ecuador son de índole bioético.

La discusión alrededor de la despenalización del aborto en Ecuador salió otra vez a la luz, luego de largos años de silencio, a partir de la propuesta presentada en noviembre del 2005 por la diputada Myriam Garcés. Se propuso una reforma de ley a los delitos de explotación sexual a menores y se contempló ampliar las causales de aborto para todos los casos de violación. Sin embargo, hasta el momento, y de acuerdo con el Art. 447 del Código Penal de la Constitución ecuatoriana, se permite el aborto terapéutico: "[...] para evitar un peligro para la vida o salud de la madre" y "[...] si el embarazo proviene de una violación o estupro cometido en una mujer idiota o demente". (Castello, 2008, p. 20).

Entendamos a la bioética como la actitud ante la vida, el respaldo moral y conductual de cómo asumimos la vida en el seno de una sociedad. La bioética se encarga del estudio de la génesis actitudinal de situaciones que comprometen la salud de los seres vivos. Por lo tanto, implica conexiones con cuestiones axiológicas (valores) psicológicas, físicas, clínicas, el respeto a las cosmovisiones (religión) y al legado político-cultural-social de una nación, todo en función de la conservación de la salud y de la vida.

Para todo aquel que tome en serio el aborto, que lo estudie y más cuando se involucra experimentalmente en el mismo, resulta más complejo entender si es prudente o no. Alrededor del aborto inducido hay muchas incógnitas que no solo tienen que ver con el paciente, supone una red de responsables: el médico, el embrión o feto, la sociedad. ¿En cuáles circunstancias el aborto inducido se podría considerar un homicidio? ¿Qué es preferible: traer una vida no deseada o no permitir que nazca? ¿Hasta qué punto de la consciencia humana no es deseada esa vida sin llegar a conocerla? ¿Cuándo es conveniente según los pilares bioéticos inducir un aborto? Estas preguntas nos invaden la mente cada vez que pensamos en el aborto inducido como fenómeno social.

De ahí que el presente tiene como propósito cognoscitivo reflexionar críticamente y desde los pilares de la bioética la ontoepistemología del aborto en Ecuador. Para ello, se requiere de una metodología que supone ideas claves del construccionismo social, un enfoque que habla de verdades con $v$ minúscula (relativas $y$ circunstanciales), convida al diálogo, exige valoración de la tradición cultural, de los compromisos con la vida social porque "todo lo que consideramos real ha sido construido socialmente. O lo que es más radical, nada es real hasta que la gente se pone de acuerdo en que lo es." (Gergen and Gergen, 2011, p. 13) y técnicas de recogida de datos como: fichajes bibliográficos y entrevistas narrativas. Lo real para nuestro ensayo es el propio problema/objeto de estudio: ¿Cómo fortalecer juicios bioéticos respecto al aborto?: una interrogante que surgió a partir de la lectura del texto: The teaching of medical ethics to medical students, escrito por Shimon M Glick en 1994, el pasado siglo y más vigente que nunca. No obstante, nos quedamos con una idea que nos revolvió el espíritu intelectual y moral del cual habló una vez Aristóteles para referirse a la virtud humana. Glick (1994), dijo: “The most caring 
doctor may be totally ignorant of academic ethics. And this should come as no surprise. Has anyone ever said about a friend or neighbour that the reason he is thoughtful and kind is that he has studied ethics?" (p. 239). La bioética como ética de lo vivo, nos conllevó a desarrollar este ensayo teniendo en cuenta la siguiente estructura (gráfico 1):

\section{Gráfico 1: Organigrama de los tópicos a tratar en el ensayo}

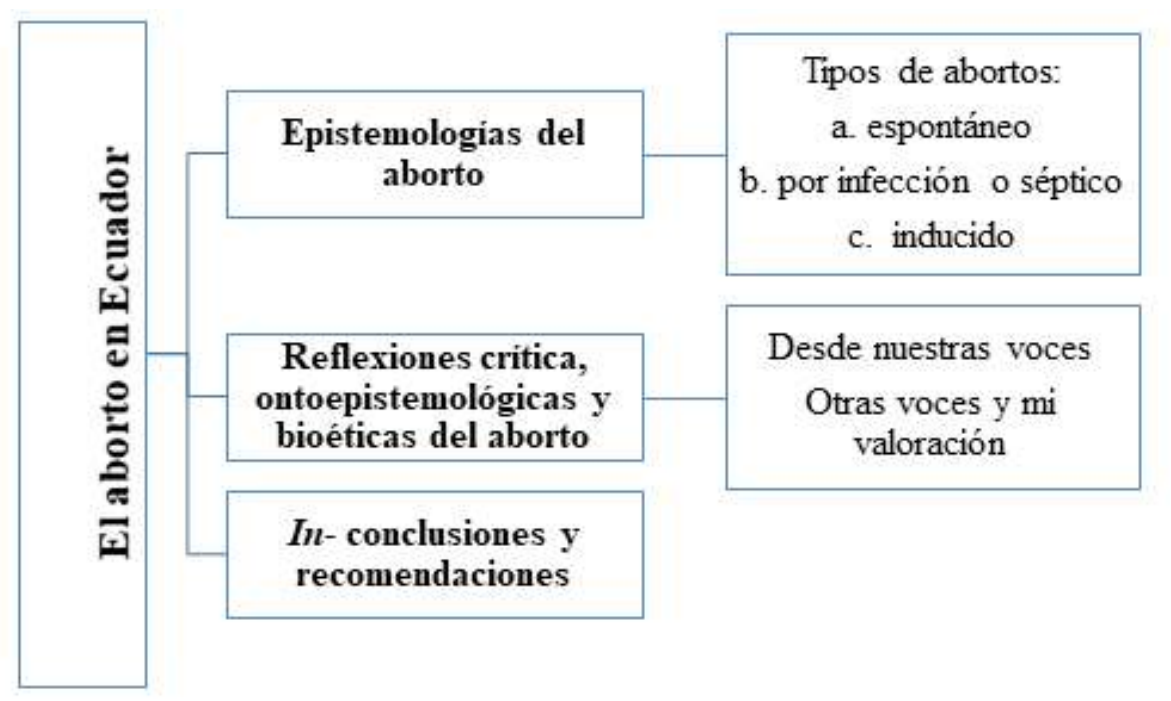

\section{DESARROLLO}

\section{1- Epistemologías del aborto}

Antes de adentrarnos en cuestiones candentes del abordo, hemos considerado necesario conceptualizar y tratar algunas tipificaciones del mismo. A sabiendas que si no entendemos lo que es un aborto, mucho menos podemos llegar a un discurso más profundo. Por lo tanto, la primera pregunta que debemos hacernos es ¿qué entendemos por aborto? y para llegar a la tipificación, ¿con qué clasificaciones de abortos vamos a trabajar?

PRÁCTICA FAMILIAR RURAL|Vol.4| No.3| Noviembre 2019 |www.practicafamiliarrural.org
El aborto, según OMS (2019), "es la expulsión o extracción de su madre de un embrión o feto de menos de 500 gramos de peso, que se alcanza a las 22 semanas" (p.1). Otros autores como Rigol y Santisteban (2014) expresaron:

El aborto es la interrupción de la gestación en las primeras 20 semanas o la expulsión del producto de la concepción que pesa menos de $500 \mathrm{~g}$. La interrupción del embarazo antes de las 10 semanas se llama aborto precoz; aquella que ocurre desde la semana 11 hasta la 20, se denomina aborto tardío. La OMS ha utilizado mucho los términos siguientes: muerte fetal precoz, cuando esta se produce antes de las 21 semanas; muerte fetal intermedia, la que tiene lugar entre las 21 y 27 semanas inclusive, y muerte fetal tardía la que ocurre a partir de las 28 semanas. Es conveniente, además, distinguir el aborto accidental, que se produce de manera aislada, del aborto a repetición o habitual, que se produce 3 veces consecutivas o más. Finalmente, es necesario diferenciar el aborto provocado, en el cual existe la intención de producirlo, del aborto espontáneo, en el que no existe ese propósito. (p. 137).

En nuestro ensayo vamos a trabajar con tres clasificaciones fundamentales: el aborto espontáneo, séptico e inducido o provocado. Haremos énfasis en el aborto provocado o inducido porque es el que históricamente ha generado más polémica bioética.

El aborto espontáneo ocurre de forma no premeditada; es decir, sin que la embarazada se percate de ello ni lo espere, esto ocurre en un $10 \%$ aproximadamente de las erróneamente menstruaciones retrasadas o profusas (Rigol y Santisteban, 2014). Existen múltiples causas que provocan abortos espontáneos y pocas son precisas ya que pueden intervenir más de una y en cada caso indistintamente. No obstante, existen dos clasificaciones generales de estas causas, una ovular y la otra materna. "En el aborto de causa ovular se produce la degeneración y muerte del embrión y su trofoblasto. En el de causa materna, el organismo de la madre impide su nutrición y el desarrollo del huevo, lo que causa su expulsión" (Rigol y Santisteban, 2014, p. 138).

El aborto por infección o séptico, constituye un problema médico de morbilidad, peligroso que pude convertirse en una causa de mortalidad materna. Este tiene que ver con condiciones econ'pomicas y sociales del paciente, así como bioético, ya que Nos atrevemos a utilizar la siguiente cita larga porque consideramos que es precisa y abarcadora. 
El aborto séptico se define como un proceso infeccioso ascendente caracterizado por una endometritis, anexitis y parametris por contaminación ovular durante las maniobras abortivas o por rotura espontánea de las membranas ovulares. Constituyen factores de riesgos el embarazo avanzado, ausencia de asepsia adecuada, dificultades técnicas en la evacuación uterina o presencia no sospechada de patógenos de transmisión sexual o de gérmenes de la flora normal potencialmente de la vagina, endocérvix, genitales externos y región perineal, que estan constituido por Enterobacterias, Pseudomonas, anaerobios como Bacteroides y Clostridium. Este último desarrolla un cuadro denominado síndrome de Mondor, situación dramática que sigue a un aborto séptico, aparece dentro de 24 a $48 \mathrm{~h}$ después del aborto provocado o espontáneo, es extremadamente grave con alta tasa de mortalidad alrededor de $60 \%$, provocado en el $80 \%$ de los casos por Clostridium perfrigens, es un síndrome toxémico-hemolítico que como resultado de la endotoxemia ocasiona hemólisis severa acompañada de trastornos de la circulación, con shock, coagulación intravascular diseminada (CID), acidosis metabólica e insuficiencia renal aguda(IRA). (Coronado et al, 2006, p.1).

El aborto provocado o inducido es aquel en el cual hay una intervención para producir una expulsión del embrión o feto. Por lo general ocurre este tipo de aborto porque fue un embarazo no deseado, porque se cree que las condiciones subjetivas y objetivas no están creadas, porque fue accidental o por una violación, etc. (eugenésico); porque se sospecha o diagnostica una malformación en el feto o algún síndrome (terapéutico).

\section{2- Reflexiones críticas, ontoepistemológicas y bioéticas del aborto}

Con anterioridad dimos a conocer algunas pistas epistemológicas relacionadas con el aborto, esas pistas conceptuales no general mayor dilema, los autores en su mayoría coinciden en qué es un aborto y los tipos que existen; lo candente está en la aceptación o no de la conducta cuando se trata de un aborto inducido, lo mismo por la intención terapéutica que por la eugenésica. Lo cierto es que ocasiona muchos dilemas y malestares en la conciencia personal y social ya que no siempre los métodos son los adecuados ni se cumple con lo estipulado por la ley en Ecuador. De ahí que abordaremos estos dos apartados con voces críticas y su relación con cuestiones ontológicas y bioéticas a través de entrevistas narrativas y diálogos.

EL aborto es un tema muy controversial a nivel mundial ya que estadísticamente ha ido en ascenso el número de personas que experimentan un aborto, ya sea por cualquiera de las formas inevitables, como abortos incompletos, completos, diferidos, sépticos, etc, como por abortos terapéuticos. Según la OMS cada año se registran 22 millones de abortos peligrosos, de los cuales 47000 provocan defunciones y cerca de 5 millones provocan complicaciones que pueden ser graves. De esta manera, el tema es de vital importancia para las grandes organizaciones mundiales de la salud ya que no solamente trae el aumento de procederes inseguros, de pérdidas humanas, sino que representa una gran pérdida de recursos y economía para los países, más frecuentemente en los desarrollados que son los que se ven más afectados.

En Ecuador que es particularmente donde tenemos nuestras vivencias según las Guías Prácticas Clínicas se registran un gran número de abortos espontáneos, en donde la mayoría de ellos ocurren en el primer trimestre del embarazo y además se ven más asociados a malformaciones y alteraciones fundamentalmente cromosómicas. Se trabaja en equipo multidisciplinario para disminuir el riesgo de complicaciones en las pacientes que lo viven, disminuyendo así un menor porcentaje de muertes que es la intención. Se ha elaborado una Guía Práctica clínica de Aborto Terapéutico con la misma finalidad de disminuir la muerte materna, este está indicado teniendo en cuenta artículos en la constitución donde se protege la vida de la mujer, en el cual se tiene presente que en todos los casos donde exista riesgo para la vida de la futura mamá se debe explicar a la pareja las posibles complicaciones, donde se debe reflejar explícitamente las ventajas y desventajas en escoger o no este procedimiento, también cuando esta una violación a una persona discapacitada. Esto es de suma importancia ya que se le da cierto protagonismo a la pareja donde ellos mismos pueden tomar su propia decisión, existiendo algoritmos de manejo, respetando siempre la decisión de la pareja, registrándose de manera adecuada el consentimiento de la paciente.

En algunos países los abortos provocados son de cierta manera tan legales que se convierten en un método anticonceptivo más, sin tener presente las grandes complicaciones que este procedimiento pudiera desenlazar, tampoco los países que lo practican se han detenido a pensar y calcular cuanta pérdida provoca este, ya que el riesgo de complicaciones y estadía hospitalaria por las mismas se eleva. Durante los últimos años se ha demostrado por el registro de las Naciones Unidas que aproximadamente el $98 \%$ de los países permiten las prácticas del aborto terapéutico cuando durante el curso del embarazo existe alguna alteración que comprometa la vida de la mujer gestante.

El artículo 150 del Código Orgánico Integral Penal ecuatoriano, publicado el 10 de febrero del 2014, establece: “El aborto practicado por un médico u otro profesional 
de salud capacitado, que cuente con el consentimiento de la mujer o su cónyuge, pareja, familiares íntimos o su representante legal, cuando ella no se encuentre en posibilidad de prestarlo, no será punible en los siguientes casos:

- Si se ha practicado para evitar un peligro para la vida o salud de la mujer embarazada y si este peligro no puede ser evitado por otros medios.

- Si el embarazo es consecuencia de una violación en una mujer que padezca discapacidad mental. (MSP, 2015, pp.12-13)

Fuera de estos casos en los últimos años se ha visto una notable incidencia de pacientes atendidos con abortos incompletos o complicaciones de los mismos, por lo que sería prudente llevar a cabo investigaciones más profundas en este sentido, ya que la causa podría estar vinculada a la práctica de estos procederes por profesionales no especializados o en sitios no seguros, donde no existen las condiciones óptimas, teniendo en cuenta que las complicaciones más frecuentes que se registran son shock séptico y shock hipovolémico. De esta manera La Organización Mundial de la Salud indica e invita a los países del mundo a dar orientación amplia acerca de la anticoncepción a las mujeres en edad fértil, la importancia de evitar embarazos no deseados, así como tener embarazos seguros, también cómo actuar ante las posibles complicaciones de estos procedimientos. Es muy importante este punto ya que asumiría desde mi pun to de vista que el programar y planificar adecuadamente un embarazo por la pareja evitaría el mayor porcentaje de complicaciones durante la gestación, así como evitaría la cantidad de abortos peligrosos.

\subsection{1- Métodos y riesgos: decisiones controversiales}

El Misoprostol es un medicamento análogo de las prostaglandinas que desencadena las contracciones uterinas, altamente eficaz en el tratamiento del aborto, fundamentalmente si este es incompleto. Tiene una elevada aceptación por los pacientes ya que ha demostrado tener mayores beneficios; por ejemplo, el costo es inferior, con una estadía hospitalaria innecesaria en muchas ocasiones, ya que si es usado en mujeres que tienen un tiempo de gestación inferior a 12 semanas no requieren hospitalización, además los efectos son similares a los de una menstruación, y evita los riesgos propios de una cirugía, en caso de elegir otro tipo de proceder. En Ecuador, Perú y Colombia, donde el aborto no es legal, se ha venido abriendo una puerta al debate sobre su comercialización por el gran uso que se le da.

PRÁCTICA FAMILIAR RURAL|Vol.4 | No.3 | Noviembre 2019 | www.practicafamiliarrural.org
Respecto a la vía de administración hasta el momento no existen evidencias potentes que indiquen elegir una vía en especial. La vía de administración del medicamento no influye en la tasa de éxito. Es muy importante siempre indicarles a las pacientes que se van a someter al procedimiento con estas tabletas que el sangrado puede ocurrir en horas o puede durar hasta dos semanas, además se acompaña de dolor tipo cólico, náuseas, malestar, el conocimiento de dichos síntomas evita la ansiedad. Además, hay algo que deben saber y es cuándo regresar al médico: si el sangrado es muy intenso, saturando una toalla en menos de una hora por dos horas consecutivas o si apareciera fiebre. (OMS, 2013)

(...) para la interrupción de un embarazo complicado por anomalías o muerte fetales en el segundo o tercer trimestre, el misoprostol vaginal es más efectivo que el misoprostol oral y es tan efectivo como las prostaglandinas tradicionalmente utilizadas y más costosas, como la PGE2, la PGF2 $\alpha$ y el gemeprost, que son más difíciles de almacenar que el misoprostol y están asociadas con más efectos secundarios. (OMS, 2010, p.1).

Estas tabletas se pueden administrar por vía vaginal, oral y/o rectal. Las mismas se vende en farmacias, son tabletas de $200 \mathrm{mcg}$. Se indican de 4 a 8 tabletas. Es aconsejable usar el misoprostol dentro de las 9 semanas a partir de la FUR. Mientras menos tiempo de gestación tenga la paciente, pues menos complicaciones. (International Women Health Coalition, 2016).

Lo ideal es no llegar a utilizar dichas pastillas, sino el condón u otro mecanismo anticonceptivo si aún no desean reproducir o no están en condiciones para ello. Hay dolor cuando comienza la expulsión, sangramiento, entre otros síntomas y signos; pero no es un método invasivo ni tan doloroso como cuando se succiona y se pinza el cuello uterino.

\section{a. Aborto por succión/aspiración uterina}

Este método es más invasivo y traumático porque consiste en poner espéculo, pinzar el cuello uterino, descomponer la estructura anatómica embrionaria con pinzas y aspirar lo que queda de producto. En ocasiones el uso de las píldoras termina con la limpieza (succión). La Dra. Dayamy Escalona León, me contó -con tristeza- la historia sobre su primer embarazo en Ecuador. Primero se atendió en la consulta de infertilidad porque tenía una endometriosis, un útero retroverso e irregularidades en la ovulación. Logró salir embarazada y a las 6 semanas tuvo un aborto espontáneo que culminó en la succión, según el especialista que la trató, para evitar restos, es 
decir, para limpiar. En tales circunstancias, el método es legal y necesario como tratamiento preventivo.

\section{b. Afectos, efectos, riesgos- daño uterino e infecciones}

Tanto el método por píldoras como la succión tienen sus efectos adversos psicológicos, sociales y físicos en la gestante. Estos afectos y efectos dependen de la causa y del propósito del aborto. En Ecuador no es bien visto por gran parte de los entes sociales un aborto "injustificado": aquel que no es terapéutico sino por decisión propia o como resultado de una experiencia irresponsable que pone en riesgo la vida de la embarazada y sacrifica al embrión o feto. Por muchos es considerado un homicidio, un atentado contra la vida de un ser indefenso.

Las personas no han concientizado el acto de violencia, muchas veces por desconocimiento de la fisiología reproductiva, no saben desde qué momento hay vida intrauterina, no saben que el útero sufre cambios durante los procedimientos invasivos. En un estudio realizado sobre el uso del misoprostol en Argentina,

para la mayoría de mujeres el aborto en sí mismo fue vivido como una menstruación normal o más intensa. Esto creó la percepción de que un aborto estaba cerca de ser "un proceso natural" y les permitió lidiar mejor con sus ansiedades y miedos (Ramos, 2016, p. 142).

En Ecuador las mujeres prefieren no opinar, más que algo ilegal es considerado un pecado mortal. Aunque sea por tratamiento existen tabúes, complejos, prejuicios que impiden estudios certeros al respecto. Esta falta de transparencia ante el fenómeno produce un incremento de abortos clandestinos que acarrean otras complicaciones como infecciones ya que las intervenciones se realizan en espacios que no cumplen con las medidas higiénicas sanitarias pertinentes. También puede ocurrir que el aborto no se haya completado y queden restos que se necrosan y producen sepsis uterina. Esto sucede, además, por los costos de la intervención. "EI aborto incompleto es una de las emergencias obstétricas de mayor demanda en el mundo entero, y los costos por su atención, a pesar de tener una estancia corta, significan un monto importante para los hospitales y las propias mujeres" (Gutiérrez y Guevara, 2019, p. 57).

\subsection{2- Legalidad y ética con respecto al aborto en el Ecuador}

a. ¿Cuándo se realiza un aborto legal en Ecuador?

PRÁCTICA FAMILIAR RURAL| Vol.4 | No.3 | Noviembre 2019 | www.practicafamiliarrural.org
En el Ecuador el aborto como problemática de salud pública no ha sido abordado en concordancia con su magnitud y se carece de estudios que permitan dar cuenta de su realidad según lo ha planteado al Comité de la CEDAW el movimiento de mujeres de ese país. A nivel penal, en ese país el aborto es tipificado como un delito contra la vida. En el código penal, no son punibles el aborto terapéutico y el eugenésico. (Lafaurie, 2005, p. 7).

Esto trae consigo que el aborto inducido sea manejado, en la mayoría de los casos, de forma clandestina; lo que puede acarrear a complicaciones como hemorragias, sepsis y hasta la muerte de las embarazadas que se someten a ello.

Se realiza de forma legal cuando el médico determina que el tratamiento para preservar la vida de la mujer depende del aborto. Por ejemplo, en una muerte fetal antes de las 22 semanas, es vital inducir el aborto en la embarazada porque

Cuando se produce la muerte fetal intrauterina, rápidamente comienza la degeneración del feto y de la placenta. En ausencia de membranas rotas e infección activa del feto, el líquido amniótico continúa siendo estéril (...) donde los productos de degeneración de proteínas sirven como un buen medio de cultivo para la contaminación bacteriana de la cavidad amniótica. (Rigol, 2014, p. 240).

\section{b. ¿Qué lleva a las mujeres realizarse un aborto?}

La respuesta a esta pregunta es compleja y complicada porque hay múltiples causas, entre las más mencionadas están:

- Embarazo no deseado: no están en condiciones para ser madres, no hubo planificación familiar, por lo tanto, fue algo accidental y ni la madre ni el padre están dispuestos porque tienen otros proyectos de vida.

- Violación: la embarazada no quiero un hijo/a de alguien que usó la fuerza o tuvo el coito en contra de su voluntad.

- Terapéutico: por muerte fetal, por malformaciones congénitas, por alteraciones cromosómicas (síndromes), etc.

Estas son las causas que conocemos de manera empírica, por lo que hemos escuchado extraoficialmente, por lo que hemos leído en otros contextos ajenos al ecuatoriano; SIN EMBARGO, la revista Redacción Médica publicó un artículo de Ortiz (2017), investigador de la UDLA, en el que plantea lo siguiente: "El 85\% de los 
abortos registrados en el Ecuador son por causas desconocidas. Esto abre la posibilidad de que parte de ellos sean autoinducidos o por procedimientos clandestinos" (p. 1). De manera que no se conoce a ciencia cierta qué lleva a las mujeres realizarse un aborto.

\section{c. Autonomía y beneficencia de la mujer}

Desde el punto de vista bioético y teniendo en cuenta los pilares de autonomía y beneficencia del paciente, el aborto inducido sigue siendo un problema latente en Ecuador. No siempre las leyes responden a principios éticos, ni a la tradición cultural imperante, tampoco a las complejas vidas de las personas. A la ley se les escapan muchas experiencias y decisiones humanas.

En Ecuador la mujer no tiene autonomía para decidir, de manera legal, por un aborto inducido que no sea terapéutico. Opinar con juicios de valor es peligroso desde el punto de vista ético respecto a este tema. La autonomía es una condición que se logra no solo porque tengas voz y voto, la autonomía depende de la madurez ética que implica responsabilidad, respeto a la vida y prudencia. Esta última supone razonar a priori a los hechos, tener visión de los efectos, controlar lo que Platón denominó "alma concupiscible", la de los seres vivo que actúan por instinto; cuya mente, más que la carne, es débil.

La beneficencia no se debe buscar en las leyes ni en los protocolos sociales sino en la libertad individual. Un bien para todos es ser precavidos ante las situaciones previsibles y ante las situaciones imprevisibles buscar la manera de solucionarlas sin causar más daño, ni comprometer otras vidas. La beneficencia, como código bioético, va de la mano con la salud integral, no la excluyente; lo que significa que el bienestar de todo ser vivo es importante.

3. In-conclusiones y recomendaciones

- El aborto inducido es el más polémico y al que le acompaña mayor cantidad de prejuicios sociales.

- El aborto en Ecuador es un tema que debe desprenderse de algunos tabúes que impiden el progreso de las ciencias de la salud.

- La prevención es la alternativa más prudente para evitar abortos inducidos injustificados.
- Dialogue con su familia, consulte al especialista y asuma la responsabilidad social que le corresponde ante un universo cuyo capitán eres tú en relación con los demás.

4. Referencias

\section{Bibliográficas}

Gergen, K. J. y Gergen, M. (2011). Reflexiones sobre la construcción social. Madrid, España: Paidós.

Castello, P. (2008). Despenalización del aborto y nuevo proyecto constitucional: un tema polémico. Iconos. Revista de Ciencias Sociales, 32, 19-23.

Shick, Sh. M. (1994). The teaching of medical ethics to medical students. Journal of medical ethics, 20, 239-243.

Rigol, O y Santisteban, A. (2014). Obstetricia y Ginecología. La Habana, Cuba: Ciencias Médicas.

Ramos, S. et al. (2016). Experiencias de mujeres con el uso de medicamentos abortivos, en un contexto de restricciones legales: el caso de Argentina. Temas de salud reproductiva, 9, 136-148.

\section{Lincográficas}

Coronado, R. et al (2006). Aborto séptico: síndrome de Mondor. Recuperado de: http://scielo.sld.cu/scielo.php?script=sci_arttext\&pid=S0138-65572006000400009

Gutiérrez, M. y Guevara, E. (2019). Controversias en el tratamiento del aborto incompleto, AMEU versus tratamiento médico con misoprostol. Recuperado de: http://www.scielo.org.pe/pdf/rgo/v61n1/a10v61n1.pdf

International Women's Health Coalition (2016). Aborto con Misoprostol Autoadministrado: Una Guía para las Mujeres. Recuperado de: https://iwhc.org/resources/aborto-con-misoprostol-autoadministrado-una-guiapara-mujeres/ 
Lafaurie, M. M. (2005). El aborto con medicamentos en América latina. Las experiencias de las mujeres en México, Colombia, Ecuador y Perú. Recuperado de:

http://clacaidigital.info/bitstream/handle/123456789/50/aborto medicamentosAL .pdf?sequence $=5$ \&isAllowed $=y$

Ministerio de Salud Pública (2013). Diagnóstico y tratamiento del aborto espontáneo, incompleto, diferido y recurrente. Guía Práctica Clínica (GPC).

Recuperado

http://instituciones.msp.gob.ec/documentos/Guias/Guia de aborto espontaneo. pdf

Ministerio de Salud Pública (2015). Atención del aborto terapéutico. Guía Práctica Clínica (GPC). Recuperado de: https://www.salud.gob.ec/wpcontent/uploads/2016/09/Aborto-terap\%C3\%A9utico.pdf
Organización Mundial de la Salud (2019). Interrupción del embarazo. Recuperado de: http://www.abortoinformacionmedica.es/category/definicion-aborto/

Organización Mundial de la Salud (2010). Misoprostol para la interrupcción del embarazo en el segundo o tercer trimestre en embarazos complicados por una anomalía fetal o después de la muerte fetal intrauterina. Recuperado de: https://extranet.who.int/rhl/es/topics/pregnancy-and-childbirth/induction-labour$\underline{4}$

Organización Mundial de la Salud (2019). Prevención del aborto peligroso. Recuperado

de: https://www.who.int/es/news-room/factsheets/detail/preventing-unsafe-abortion

Ortiz, E. (2017). El 85\% de los abortos registrados en el Ecuador son por causas desconocidas. Recuperado de: https://www.redaccionmedica.ec/secciones/saludpublica/el-85-de-los-abortos-registrados-en-el-ecuador-son-de-causasdesconocidas -90319 